\title{
Brain MRI in Children with Mild Traumatic Brain Injury and Persistent Symptoms in Both Sports- and Non-sports-related Concussion
}

\author{
Alcy R. Torres ${ }^{1}$, Zakir I. Shaikh ${ }^{2}$, Wilson Chavez ${ }^{3}$, John E. Maldonado ${ }^{4}$ \\ 1. Pediatrics, Boston University School of Medicine, Boston, USA 2. Pediatrics, Surat Municipal Institute of Medical \\ Education and Research, Surat, IND 3. Radiology, Boston Medical Center, Boston, USA 4. Pediatrics, Pontifical Catholic \\ University of Ecuador, Quito, ECU
}

Corresponding author: Alcy R. Torres, artorres@bu.edu

\begin{abstract}
Aim: To evaluate the utility of magnetic resonance imaging (MRI) in children with mild traumatic brain injury (mTBI), persistent post-concussive syndrome (PPCS), and normal neurologic examination.

Materials and methods: This was a retrospective review of pediatric patients, who were evaluated in a Pediatric Concussion Clinic between August 2013 and November 2018, with documented persistent postconcussive symptoms, normal neurological examination, and available brain MRI.
\end{abstract}

Results: In our analysis of 86 cases we found seven MRI studies with abnormal findings, but none were clinically significant.

Conclusion: We conclude that MRI has a low diagnostic yield in this population, and based on these results we recommend that clinicians should avoid ordering MRI studies in this group. Further research is necessary to validate these results in larger and prospective studies.

Categories: Neurology, Pediatrics

Keywords: pediatric concussion, postconcussion syndrome, magnetic resonance imaging, mild traumatic brain injury, brain mri

\section{Introduction}

The aim of this study was to demonstrate the utility of brain magnetic resonance imaging (MRI) in children (aged 2-18 years) with mild traumatic brain injury (mTBI) and persistent post-concussive syndrome (>28 days) but with a normal neurological examination. We seek to identify abnormal brain imaging in these patients to assess the utility of neurological imaging in this group.

Received 01/07/2019

Review began 01/13/2019 Review ended 01/18/2019 Published 01/22/2019

๑) Copyright 2019

Torres et al. This is an open access article distributed under the terms of the Creative Commons Attribution License CC-BY 3.0., which permits unrestricted use, distribution, and reproduction in any medium, provided the original author and source are credited.
Traumatic brain injury (TBI) is one of the leading causes of hospital visits in the United States. In the last study cited by the Centers for Disease Control and Prevention (CDC) in 2013, there were approximately 640,000 TBI-related emergency department (ED) visits, 18,000 TBI-related hospitalizations, and 1,500 TBIrelated deaths in children aged 0-14 years in the USA [1]. In 2012, approximately 325,000 ED visits resulted from sports and recreation-related mTBI [2]. Among the pediatric population with mTBI, $82 \%$ initially visited primary care, $5 \%$ searched for specialty care, and $12 \%$ were assisted at the ED [3,4].

TBI can be classified in a number of ways but traditionally the classification is based on clinical severity and duration of symptoms, and characteristics and location of the injury [5]. The severity of TBI is most commonly quantified by the Glasgow Coma Scale (GCS). A GCS score of 13-15 is considered mild injury, 912 is considered moderate injury, and eight or less is considered severe TBI [6].

There are different mechanisms of traumatic brain injury occurring in sports, war, and life. These mechanisms are closed head traumatic brain injury, blast-related brain injury, and penetrating brain injury. Those share many common features in the potentiation of injury through secondary injury mechanisms and physical and neuropsychological outcome. However, significant differences in pathophysiological pathways, gross neurologic damage, and the time scale of neurodegeneration exist, which have been studied using different methods [7].

Mild traumatic brain injury (mTBI) is defined as "an acute brain injury resulting from mechanical energy to the head from external physical forces”. The operational criteria for clinical identification include:

- One or more of the following: confusion or disorientation, loss of consciousness (LOC) for 30 minutes or less, post-traumatic amnesia for less than 24 hours, and/or other transient neurologic abnormalities such as focal signs, seizure, and intracranial lesion not requiring surgery. 
- These manifestations of mTBI must not be due to drugs, alcohol, medications, caused by other injuries or treatment for other injuries (eg, systemic injuries, facial injuries, or intubation), caused by other problems (eg, psychological trauma, language barrier, or coexisting medical conditions), or caused by penetrating craniocerebral injury [8].

More than $75 \%$ of children with traumatic brain injuries attended to in the emergency department in the United States are defined as mild [9], but the numbers seeking care are rising dramatically [10] due to increased public awareness about concussion, improved training of clinicians in concussion diagnosis, and a legislation in all 50 states requiring healthcare provider clearance prior to returning to play [3,4]. Traumatic brain injury is the leading cause of death and disability in children older than one year [11]. The concerns about its effects on the developing brain have led to a large number of recent studies examining pediatric concussion [12]. Rivara et al. found that an approximate of $14 \%$ of children with mTBI experienced disability, defined as the use of specialized medical and educational services [13].

Fortunately, almost $90 \%$ of concussions are transient, with symptoms resolving within four weeks. However, a minority of patients remain symptomatic several months post injury [14]. The most common symptoms encountered at presentation are headaches, dizziness, and taking longer to think whereas sleep disturbance, frustration, forgetfulness, and fatigue are the symptoms most likely to develop during the follow-up period and that had not initially been present [12].

Symptoms persisting beyond 28 days are referred to as persistent post-concussion syndrome (PPCS) and can have serious adverse effects, resulting in school absenteeism, impaired academic performance, depressed mood, loss of social activities, and lower quality of life [15]. These symptomatic patients are usually the population seen in the clinic after the event, and the persistence of symptoms despite their normal neurological examination raises the concern of getting neuroimaging.

There have been some studies about brain imaging in patients with persistence of symptoms more than two weeks after a concussion, which showed low diagnostic yield and had poor cost-effectiveness. One such study, done in a smaller cohort and which included other different imaging modalities, determined that neuroimaging should not be repeated beyond the acute phase. Though head computed tomography (CT) had the relatively highest yield among the modalities, the potential effect of radiation exposure must be considered. MRI, a non-radiation method, can detect post-traumatic encephalomalacia, reactive gliosis, hemosiderin deposits, and microbleeds; however, these typically only accompany more severe forms of TBI [14].

Mild-to-moderate TBI-related services in children averaged $\$ 77.9$ million per year in healthcare costs, with an average expenditure of $\$ 1,044$ per capita in which $\$ 166$ were TBI-specific services and $\$ 878$ were for general healthcare [16].

\section{Materials And Methods}

We conducted a single-center retrospective review of electronic medical records and neuroimaging (MRI) studies for all patients with mild traumatic brain injury at Boston Medical Center from 2013 to 2018. The inclusion criteria were as follows: 1) age $2-18$ years; 2 ) mTBI: GCS score of 13-15; and 3) normal neuro examination during ED evaluation after the episode and in a clinic a few days later. The exclusion criteria were as follows: 1) children with severe injury mechanisms defined by fall $>0.9 \mathrm{~m}$ [3 feet]; head struck by high impact object; motor vehicle collision with patient ejection, death of another passenger, or rollover; pedestrian or bicyclist without helmet struck by a motorized vehicle; 2) penetrating trauma, known brain tumors, pre-existing neurological disorders complicating assessment; 3) pre-existing neuroimaging finding before event.

\section{Data collection and analysis}

Provider consultation notes, MRI findings, and scanned outside hospital documentation were collected and analyzed via our institution's electronic medical record system. We collected data in three categories: 1) demographic variables, 2) past medical history, and 3) timing of MRI-only imaging obtained >four weeks after the event was included. A board-certified pediatric neurologist with the Symptom Evaluation of Children with the Sports Concussion Assessment Tool 5 (SCAT5®) determined clinically relevant findings [17]. A faculty neuroradiologist identified positive findings on MRI.

The analysis took into consideration any symptoms related to the trauma at the time of evaluation in the clinic after the event. Post-concussion symptoms were investigated using the symptom evaluation list of Children SCAT-3 \& SCAT-5. Patients and their family responded to the questionnaire after the trauma.

All data was irreversibly made anonymous and this study was conducted according to the guidelines of the ethical committee of our institution. The institutional review board approved this study, with a waiver of 


\section{Cureus}

informed consent. The study protocol complied with the Health Insurance Portability and Accountability Act.

\section{Results \\ Demographics}

The mean age of all patients was 14.17 years with a range between six and 18 years (Table 1 ). The study included 46 females (53.49\%) and 40 (46.51\%) males. Most patients were of the $12-18$ years age group.

Table 1. Demographic characteristics of participants

\begin{tabular}{|c|c|c|}
\hline & Frequency & Percentage \\
\hline Total (N) & 86 & $100 \%$ \\
\hline \multicolumn{3}{|l|}{ Age group } \\
\hline $2-5$ & 0 & $0 \%$ \\
\hline 6-11 & 17 & $19.77 \%$ \\
\hline $12-18$ & 69 & $80.23 \%$ \\
\hline Age mean & 14.17 & \\
\hline Number of patients & 86 & \\
\hline \multicolumn{3}{|l|}{ Gender } \\
\hline Male & 40 & $46.51 \%$ \\
\hline Female & 46 & $53.49 \%$ \\
\hline
\end{tabular}

\section{TABLE 1: Demographic characteristics of participants}

In all the patients with mild TBI, the most common symptom was headaches (88.37\%), followed by memory, attention, and concentration disturbances (37.21\%), and, lastly, visual disturbances (e.g. blurry vision, photosensitivity, photophobia) $36.05 \%$. But, most of the children had more than one symptom (Table 2).

Table 2. Signs and symptoms in patients

$\begin{array}{lcc}\text { Symptoms } & \text { Frequency } & \text { Percentage } \\ \text { Headaches } & 76 & 88.37 \% \\ \text { Memory, attention, concentration disturbances } & 32 & 37.21 \% \\ \text { Visual disturbances } & 31 & 36.05 \% \\ \text { Dizziness } & 28 & 32.56 \% \\ \text { Auditory disturbances } & 14 & 16.28 \% \\ \text { Sleep disturbances } & 10 & 11.63 \% \\ \text { Nausea } & 8 & 9.30 \%\end{array}$

\section{TABLE 2: Signs and symptoms in patients}

Only 11 (12.79\%) of the patients lost consciousness at the time of injury, five of them for less than one minute. Thirty-three (38.37\%) of the patients had a sport-related concussion, where football was involved in most cases (seven) (8.14\%), followed by martial arts (five cases) (5.81\%). Fifty-three (61.63\%) were not sport related, of which 17 (19.77\%) were due to a motor vehicle accident, 17 (19.77\%) were due to a fall, seven $(8.14 \%)$ were due to a head strike, and seven (8.14) were due to an assault. 


\section{Cureus}

The most common mechanism of injury was direct impact (88.37\%) as a result of a collision with other objects. Six (6.98\%) of the patients had post-traumatic amnesia, of which one (1.16\%) was anterograde, two (2.33\%) were retrograde, and three (3.49\%) had amnesia of the event.

The history of a prior concussion was recorded as: none (62.79\%), one (24.42\%), two (5.81\%), three (2.33\%), and more than four (4.65\%). In our study, there was no relation between multiple concussions and abnormal MRI results. Most of the patients had an immediate onset (76.74\%) of symptoms.

Some patients (75) $(87.21 \%)$ responded to symptomatic treatment used for their most prominent symptoms, which include pharmacologic and non-pharmacologic therapies (Table 3).

Table 3. Clinical characteristics of patients

Characteristics

Frequency

Percentage

Type of injury

Sport-related

33

$38.37 \%$

Football

7

$8.14 \%$

Martial arts

5

$5.81 \%$

Gymnastics

3

$3.49 \%$

Softball

3

$3.49 \%$

Cheerleading

2

$2.33 \%$

Non-sport related

53

$61.63 \%$

Motor vehicle accident

17

$19.77 \%$

Fall

17

$19.77 \%$

Head strike

7

$8.14 \%$

Assault

7

$8.14 \%$

Mechanism of injury

Direct impact

$88.37 \%$

Acceleration/deceleration

6

$6.98 \%$

Not specified

4

$4.65 \%$

Loss of consciousness during event

11

$12.79 \%$

Average duration of loss of consciousness

$<1$ minute

5

$5.81 \%$

$>1$ minute

6

$6.98 \%$

Post-traumatic amnesia

6

$6.98 \%$

About the event

Retrograde

2

$2.33 \%$

Anterograde

$1.16 \%$

Prior concussion

None

$62.79 \%$

1

21

$24.42 \%$

2

5

$5.81 \%$

3

2

$2.33 \%$

$>4$

$4.65 \%$

Prior neuro-psychologic disorder

39

$45.35 \%$ 


\section{Cureus}

\begin{tabular}{lll} 
Attention deficit hyperactive disorder & 12 & $13.95 \%$ \\
Depression & 8 & $9.30 \%$ \\
Anxiety & 6 & $6.98 \%$ \\
Onset of symptoms & & \\
Immediate & 66 & $76.74 \%$ \\
Gradual & 20 & $23.26 \%$ \\
Response to symptomatic treatment & & \\
Positive response & 75 & $87.21 \%$ \\
\hline
\end{tabular}

TABLE 3: Clinical characteristics of patients

\section{Neuroimaging}

Out of the 1275 patients who visited the clinic, 255 patients had PPCS, and MRI was obtained in 86 of these patients with PPCS (33.73\%). Of these 86 patients, 14 (16.28\%) had prior imaging in the acute phase that was negative and subsequently repeated during the chronic phase of their PPCS course. The indication to obtain the MRI was only prolonged persistent symptoms.

Among the 86 patients with MRI, seven patients had positive imaging findings, but none were clinically significant to alter the course or the medical management (Tables 4-5).

Table 4. Neuroimaging findings

Trauma-related

Non-trauma-related

MRI scan ordered post-concussion

Timing of MRI after event

28 days -3 months

3 months -6 months

$>6$ months
86

86

Frequency

Percentage

32

$37.21 \%$

29

$33.72 \%$

$29.07 \%$

\section{TABLE 4: Neuroimaging findings}




\section{Cureus}

Table 5. Neuroimaging abnormalities

Case Findings

1 Irregularly shaped T2/FLAIR (T2-weighted and fluid-attenuated inversion recovery) hyperintensity areas in the right frontal subcortical white matter

2 Scattered tiny punctate regions of T2/FLAIR hyperintensity in the frontal lobes

$3 \quad$ Nonspecific foci of FLAIR hyperintensity in the left frontal lobe

$4 \quad$ Cluster of small cysts with mild surrounding gliosis in the right periatrial region

$5 \quad$ Nonspecific ovoid T2/FLAIR hyperintense focus within the left posterior temporal lobe

6 Atretic parietal cephalocele with a persistent falcine sinus and partial absence of the straight sinus

$7 \quad$ Numerous subcortical white matter FLAIR hyperintensities

TABLE 5: Neuroimaging abnormalities

\section{Discussion}

In the span of five years, we evaluated 1275 concussion patients of whom 255 had PPCS. MRI was ordered in 86 (33.73\%) patients but none had clinically significant findings. MRI studies require time, are expensive, use hospital resources, and might cause psychological stress for the patient and their family [16,18,19]. We conducted a retrospective study on this cohort to assess the utility of MRI and determine the need for its use.

In our study we found only seven (8.4\%) abnormal MRI; however, none of the MRI had any clinical relevance. In those patients with positive findings, further diagnostic tests or interventional treatment were not required, and the management was not modified because of the imaging results. Requesting imaging in these patients did not add value and had a low diagnostic yield.

In 2015 Morgan et al. studied 19 patients who had MRI and only one had findings that were not considered clinically significant [14]. Although this series was very small, he concluded that brain imaging should only be ordered in very specific rare occasions. Results from our study are similar to the Morgan series and corroborate the low yield of MRI in children with persistent post-concussive symptoms. The abnormal MRI value that we found in our cohort is low as in the mentioned study [14]. In 2017 Rose et al. studied 134 patients who underwent MRI evaluations, and $16.4 \%$ were found to have incidental abnormalities, a rate slightly higher than the $10.6 \%$ cited among healthy youth [20]. Incidental findings can prompt additional imaging and another testing, further increasing healthcare costs. The imaging readings can also be a source of stress and anxiety for families. So, they conclude that a better understanding of the diagnostic yield and the therapeutic benefits of neuroimaging is needed to guide clinical decision-making when post-concussion symptoms persist. A similar study was done by Bonow et al., where a large database of sports concussion in the pediatric population with persistent symptoms was explored. They studied MRI reports of 427 pediatric patients and only $0.5 \%$ had findings compatible to the traumatic episode; 61 patients had abnormal MRI, but the findings were unrelated to trauma and without any clear clinical relevance. In comparison to the above-mentioned studies, our research was different as it focused on both sports- and non-sports-related concussions and included only those patients with a normal neurological examination [21].

The estimated average list price for a single brain MRI nationwide was $\$ 2625$ per study. Among the 86 patients with PPCS who received MRIs, we estimated a total billed cost of $\$ 225,750$. The cost of one positive MRI finding was $\$ 32,250$. We used New Choice Health for the estimated cost of brain MRI [22], a resource that has been cited in previous literature.

MRI has equal sensitivity in detecting acute changes and better sensitivity than head CT in detecting some anomalies, such as cerebral edema, hemosiderin deposition, and minor cerebral contusion, and avoids the substantial radiation exposure that accompanies head CT [23]. Brain MRI can detect subtle signs of traumatic injury that may influence concussion management decisions when symptoms persist (e.g. return to play and retirement from a high-risk sport), yet a correlation between MRI findings and clinical outcomes has not been well-established. Scanty data exists about the clinical utility of MRI in patients with persistent post-concussion symptoms and the purpose of our study is to contribute more information about this controversial topic in children with mild traumatic brain injury and persistent symptoms in both sports- and non-sports-related concussion.

When present on clinic day MRI appears to have been the preferred imaging modality for late-acute and sub- 
acute cephalalgic symptoms. In our study, MRI was the method of choice to further evaluate patients with persistent post-concussive syndrome. CT is generally performed during the acute post-concussion period, while the mean time to MRI is nearly 40 days following injury in our study and others.

There may also be the possibility to predict the development of persistent post-concussion symptoms in children presenting to the emergency department, although a clinical score developed had modest discrimination to estimate persistent post-concussive syndrome risk in those patients. However, features such as female sex and older age are associated with prolonged recovery in children and adults [15].

Following the Pediatric Emergency Care Applied Research Network (PECARN) rules that guide the decisions in the ED in children with mTBI, a study found that doctor visits following mild TBI were significantly more frequent within patients from the hospitalized group than the ambulatory group [24].

The positive findings on MRI in our population do not explain the persistence of symptoms. However, a study shows that parenchymal lesion on MRI can reflect greater risk for brain insult and predict higher levels of PPCS within the MTBI [25]. Our results are probably not related because we did not find major neurological imaging changes that can alter the course of the recovery from a mild traumatic brain injury but more importantly because our subjects had normal neurological examinations. In addition to clinic day symptoms, prior concussions and continued active participation at the time of injury predicted MRI utilization.

It is also important to consider the psychological factors when deciding to order further studies in a patient with mTBI and post-concussive symptoms. Studies have suggested that psychological factors may play some role, whereby obtaining CT or MRI scans changed the patients' perceptions about their injuries and led to longer symptom recoveries [20].

The limitation of the study, in general, is its retrospective design. There is also the fact that we recorded the post-concussion symptoms using a questionnaire, leading to subjective information.

\section{Conclusions}

In our population of pediatric patients with a history of sports- and non-sports-related concussion and postconcussive syndrome, a brain MRI was of low yield diagnosis if they had a normal neurologic examination. Further research is necessary to validate our results in larger and prospective studies.

\section{Additional Information \\ Disclosures}

Human subjects: Consent was obtained by all participants in this study. Integrated Network for Subject Protection In Research II issued approval H-37677. Animal subjects: All authors have confirmed that this study did not involve animal subjects or tissue. Conflicts of interest: In compliance with the ICMJE uniform disclosure form, all authors declare the following: Payment/services info: All authors have declared that no financial support was received from any organization for the submitted work. Financial relationships: All authors have declared that they have no financial relationships at present or within the previous three years with any organizations that might have an interest in the submitted work. Other relationships: All authors have declared that there are no other relationships or activities that could appear to have influenced the submitted work.

\section{References}

1. Taylor CA, Bell JM, Breiding MJ, Xu L: Traumatic brain injury-related emergency department visits, hospitalizations, and deaths-United States, 2007 and 2013. MMWR Surveillance Summ. 2017, 66:1-16. 10.15585/mmwr.ss6609a1

2. Coronado VG, Haileyesus T, Cheng TA, et al.: Trends in sports- and recreation-related traumatic brain injuries treated in US emergency departments: The National Electronic Injury Surveillance System-All Injury Program (NEISS-AIP) 2001-2012. J Head Trauma Rehabil. 2015, 30:185-197. 10.1097/HTR.0000000000000156

3. Arbogast KB, Curry AE, Pfeiffer MR, et al.: Point of health care entry for youth with concussion within a large pediatric care network. JAMA Pediatrics. 2016, 170:e160294. 10.1001/jamapediatrics.2016.0294

4. Setnik L, Bazarian JJ: The characteristics of patients who do not seek medical treatment for traumatic brain injury. Brain Injury. 2007, 21:1-9. 10.1080/02699050601111419

5. Pervez M, Kitagawa RS, Chang TR: Definition of traumatic brain injury, neurosurgery, trauma orthopedics, neuroimaging, psychology, and psychiatry in mild traumatic brain injury. Neuroimaging Clin NA. 2018, 28:1-13. 10.1016/j.nic.2017.09.010

6. McKee AC, Daneshvar DH: Chapter 4: the neuropathology of traumatic brain injury . Handb Clin Neurol. 2015, 127:45-66. 10.1016/B978-0-444-52892-6.00004-0

7. Bauer D, Tung ML, Tsao JW: Mechanisms of traumatic brain injury. Semin Neurol. 2015, 35:14-22. 10.1055/s-0035-1549095

8. Kristman VL, Godbolt AK, Salmi LR, et al.: Methodological issues and research recommendations for prognosis after mild traumatic brain injury: results of the international collaboration on mild traumatic 
brain injury prognosis. Arch Phys Med Rehabil. 2014, 95:S265-S277. 10.1016/j.apmr.2013.04.026

9. Babcock L, Byczkowski T, Wade SL, Ho M, Mookerjee S, Bazarian JJ: Predicting postconcussion syndrome after mild traumatic brain injury in children and adolescents who present to the emergency department. JAMA Pediatr. 2013, 167:156-161. 10.1001/jamapediatrics.2013.434

10. Yeates KO, Beauchamp M, Craig W, et al.: Advancing Concussion Assessment in Pediatrics (A-CAP): a prospective, concurrent cohort, longitudinal study of mild traumatic brain injury in children: protocol study. BMJ Open. 2017, 7:e017012. 10.1136/bmjopen-2017-017012

11. Holmes JF, Borgialli DA, Nadel FM, et al.: Do children with blunt head trauma and normal cranial computed tomography scan results require hospitalization for neurologic observation?. Ann Emerg Med. 2011, 58:315322. 10.1016/j.annemergmed.2011.03.060

12. Eisenberg MA, Meehan WP, Mannix R: Duration and course of post-concussive symptoms . Pediatrics. 2014, 133:999-1006. 10.1542/peds.2014-0158

13. Rivara FP, Koepsell TD, Wang J, et al.: Incidence of disability among children 12 months after traumatic brain injury. Am J Public Health. 2012, 102:2074-2079. 10.2105/AJPH.2012.300696

14. Morgan CD, Zuckerman SL, King LE, Beaird SE, Sills AK, Solomon GS: Post-concussion syndrome (PCS) in a youth population: defining the diagnostic value and cost-utility of brain imaging. Childs Nerv Syst. 2015, 31:2305-2309. 10.1007/s00381-015-2916-y

15. Zemek R, Barrowman N, Freedman SB, et al.: Clinical risk score for persistent postconcussion symptoms among children with acute concussion in the ED. JAMA. 2016, 315:1014-1025. 10.1001/jama.2016.1203

16. Brener I, Harman JS, Kelleher KJ, Yeates KO: Medical costs of mild to moderate traumatic brain injury in children. J Head Trauma Rehabil. 2004, 19:405-412.

17. Davis GA, Purcell L, Schneider KJ, et al.: The Child Sport Concussion Assessment Tool 5th Edition (Child SCAT5): background and rationale. Br J Sports Med. 2017, 51:859-861. 10.1136/bjsports-2017-097492

18. Thukral BB: Problems and preferences in pediatric imaging. Indian J Radiol Imaging. 2015, 25:359-64. 10.4103/0971-3026.169466

19. Raschle N, Zuk J, Ortiz-Mantilla S, et al.: Pediatric neuroimaging in early childhood and infancy: challenges and practical guidelines. Ann N Y Acad Sci. 2012, 1252:43-50. 10.1111/j.1749-6632.2012.06457.x

20. Rose SC, Schaffer CE, Young, McNally KA, Fischer AN, Heyer GL: Utilization of conventional neuroimaging following youth concussion. Brain Inj. 2017, 31:260-266. 10.1080/02699052.2016.1235285

21. Bonow RH, Friedman SD, Perez FA, et al.: Prevalence of abnormal magnetic resonance imaging findings in children with persistent symptoms after pediatric sports-related concussion. J Neurotrauma. 2017, 34:27062712. 10.1089/neu.2017.4970

22. Brain MRI cost and procedure comparison. (2018). Accessed: January 8, 2019: https://www.newchoicehealth.com/procedures/brain-mri.

23. Toledo E, Lebel A, Becerra L, et al.: The young brain and concussion: imaging as a biomarker for diagnosis and prognosis. Neurosci Biobehav Rev. 2013, 36:1510-1531. 10.1016/j.neubiorev.2012.03.007

24. Wendling-Keim DS, König A, Dietz HG, Lehner M: Ambulatory or inpatient management of mild TBI in children: a post-concussion analysis. Pediatr Surg Int. 2016, 33:249-261. 10.1007/s00383-016-4021-z

25. Taylor HG, Dietrich A, Nuss K, et al.: Post-concussive symptoms in children with mild traumatic brain injury. Neuropsychology. 2010, 24:148-159. 10.1037/a0018112 